\title{
22nd International Conference on Digital Audio Effects DAFx 2019 (2-6 September 2019, Birmingham, United Kingdom)
}

\author{
Ryan Stables ${ }^{1, *}$, Jason Hockman ${ }^{1}$ and Vesa Välimäki ${ }^{2}$ (1) and Federico Fontana ${ }^{3}$ \\ 1 Digital Media Technology Lab, Birmingham City University, Birmingham B4 7XG, UK; \\ jason.hockman@bcu.ac.uk \\ 2 Acoustics Lab, Department of Signal Processing and Acoustics, Aalto University, FI-02150 Espoo, Finland; \\ vesa.valimaki@aalto.fi \\ 3 HCI Lab, Department of Mathematics, Computer Science and Physics, University of Udine, IT-33100 Udine, \\ Italy; federico.fontana@uniud.it \\ * Correspondence: ryan.stables@bcu.ac.uk
}

Received: 15 December 2019; Accepted: 23 January 2020; Published: 5 February 2020

\begin{abstract}
This meeting report gives an overview of the DAFx 2019 conference held in September 2019 at Birmingham City University, Birmingham, UK. The conference had the same theme as this special issue: digital audio effects. In total, 51 papers were presented at DAFx 2019 either in oral or in poster sessions. The conference had 157 delegates, almost half from industry and the rest from universities around the world. As the number of submissions and participants remains sufficiently high, it is planned that the DAFx conference series will be continued every autumn.
\end{abstract}

Keywords: Acoustics; audio signal processing; real-time algorithms; artificial intelligence; machine learning; music

\section{Introduction}

The 22nd International Conference on Digital Audio Effects was held at Birmingham City University, in Birmingham City Centre, UK. The event was held over five days from Monday 2nd to Friday 6th September 2019. The first day of the scientific program was allocated to tutorial sessions, in which four technical subjects were covered with a broader scope compared to the paper sessions. The three days running from 3rd to 5th September comprised the main track of the scientific program, in which talks, posters and keynotes were delivered. For the final day of the conference, the 5th Workshop on Intelligent Music Production (WIMP5) was held as a satellite event, which shared a similar format to the main track.

To promote diversity at the conference, the organizing committee introduced a code of conduct, which was intended to promote a culture of inclusivity. The conference also offered four inclusivity grants, which were aimed at helping participants who would struggle to attend for financial reasons, and to promote diversity.

\section{Organizing Committee}

The DAFx 2019 organizing committee (shown in Table 1) consisted of academics and industry professionals from six different organizations. The committee managed the organization of the conference from the event's logistics to the scientific program. Major contributions were also made by the organizers of previous conferences, particularly the teams led by Guilherme Campos (University of Aveiro, Portugal), and Stefan Bilbao (University of Edinburgh, UK) who provided materials and guidance for the event. 
Table 1. The DAFx 2019 Organizing Committee.

\begin{tabular}{lll}
\hline Role & Name & Affiliation \\
\hline General chair & Ryan Stables & Birmingham City University \\
General chair & Jason Hockman & Birmingham City University \\
Paper committee chair & David Moffatt & University of Plymouth \\
WIMP5 general chair & Nicholas Jillings & Birmingham City University \\
Sponsorship chair & Brecht De Man & Semantic Audio Labs \\
Poster session chair & Maciej Tomczak & Birmingham City University \\
Sponsorship & Carl Southall & Music Tribe \\
Merchandise & Jake Drysdale & Birmingham City University \\
External program lead & Sean Enderby & Birmingham City University \\
Technical lead & Matthew Cheshire & Birmingham City University \\
Program committee & Aglaia Foteinou & University of Wolverhampton \\
Program committee & Hsein Kew & Birmingham City University \\
Program committee & Xueyang Wang & University of Warwick \\
\hline
\end{tabular}

\section{Statistics}

In total, 51 papers were accepted for publication at DAFx 2019. Of these, 28 were allocated to oral presentations, and 23 were allocated to poster presentations. These allocations were based on the relevance of the paper to a session, and equivalent importance was given to both modes of presentation.

Including the organizing committee, 157 attendees registered for the event. This year, there was a relatively even distribution of attendees across institution type, with $40 \%$ of delegates being from industry, $27 \%$ students, and $33 \%$ of delegates registered as academics. Like similar audio conferences [1], there was a gender imbalance with only $8 \%$ of the registered delegates being female. This will likely change in years to come through the efforts of organizing committees to promote inclusivity.

While DAFx is primarily located in Europe, the conference has an international reach. This year, attendees travelled to the conference from 14 different countries, $75 \%$ of delegates were based in Europe, $20 \%$ in North America, and around 5\% in Asia. The most common locations by country were UK (24\%), Germany (22\%), USA (22\%), and France (12\%).

\section{General Directions}

The oral sessions at DAFx 2019 were partitioned into themes, consisting of:

- Digital Signal Processing

- $\quad$ Sound Synthesis

- Virtual Analog

- Spatial Audio

- Deep Learning for Sound Synthesis

- Deep Learning for Audio Effects

As the session titles suggest, this year there was a notable subset of the papers which focused on the use of machine learning methods to approximate audio effects and synthesis parameters. This use of machine learning is a noticeable trend in audio technology, and is confluent with most other fields that use digital technologies. The DAFx conferences have also been known for their emphasis on virtual analog modeling. This year, a large subset of the papers focused on modeling black and grey box systems, with several contributions on physical modeling. Interestingly, there was a cross-over this year, whereby machine learning methods were used to approximate the parameters of complex virtual models.

As this was an audio processing conference, most presentations included audio examples, which helped the audience to get a clear idea of the goals of the presented work and of the obtained sound 
quality. Some poster presenters had brought a computer running a real-time demo of their work, which could be enjoyed using headphones.

\section{Tutorials}

Four tutorial sessions were delivered during the first day of the conference. These covered a range of related topics in more detail. This year, the tutorials were selected to coincide with the subjects of interest to the community.

The first tutorial Creative Applications of Music and Audio Research, given by Amélie Anglade and Ryan Groves discussed a range of AI-based solutions, which are being used in industry for musical purposes. The focus of the talk was on the trend of creativity in machine learning systems, which the authors were able to discuss at length due to their background in music information retrieval.

The second tutorial Creative Use of Audio Plugins in a Mix: A Live Mixing Session with a Grammy Award Winner, given by Steven Roessner was an interactive mixing session, in which Roessner discussed at length the creative use of audio effects in the mixing and master process. He discussed a range of concepts when mixing and mastering music, and provided insight into the mix that won the Grammy award.

The third tutorial Room Response Equalization, given by Stefania Cecchi (Università Politecnica delle Marche, Ancona, Italy), explored the use of automated equalizer design based on features of the acoustic listening environment. The talk provided a history of the field, and a critical review of the most commonly used advanced DSP methods. The presentation included excellent audio examples demonstrating the improvement obtained with some of the methods. Cecchi and her colleagues recently published a review article on the same topic [2].

The final tutorial of the session, Principles of Wave Digital Filter Modeling for Virtual Analog: A Tutorial, given by Kurt Werner (SARC, Queen's University Belfast, UK), gave a comprehensive guide to the use of Wave Digital Filters. These are fundamental methods in the field of virtual analog modeling, which were described in a great level of detail, from early developments of circuit models, to recent additions such as multiple non-linearities and complex topologies [3,4].

\section{Keynote Speakers}

Three keynote speakers presented their work at the conference. On the first day of the scientific program, Jesse Engel of Google Research covered the work of the Magenta Project. This large scale, open source research project encompasses the work of several engineers at Google and aims to generate music and art using machine learning. The keynote covered a range of techniques for content generation, including an LSTM-based method for generating musical sequences and a VAE-based model for learning long-term musical structure.

On the second day of the scientific program, Paul Weir of Earcom discussed his work on generative and procedural audio systems. He discussed his work on the notorious console game No Man's Sky, in which several aspects of the in-game universe were procedurally generated. Several systems that were used for sound generation were discussed in detail.

On the final day of the scientific program, Masataka Goto of AIST Japan presented his work on intelligent music interfaces, which spans several decades. He discussed the origins of beat tracking, whereby he was trying to track the pulse of musical signals to make cartoon robots dance. The keynote then covered several recent projects such as Songle Sync [5]: a platform that allows a large number of internet-connected devices to be synchronized to music, and TextAlive [6]: an authoring tool for kinetic typography which synchronizes to music. An important message from the keynote was that unforeseen use-cases often emerge from interesting research projects.

\section{Best Papers}

This year three best paper awards were given out, two of which were for general paper quality, and one was for the best student paper. The criteria were based on the overall paper scores from 
reviewers, and a small panel of committee members. Interestingly, this year the leading papers were all related to signal processing methods.

Non-Iterative Phaseless Reconstruction from Wavelet Transform Magnitude, by Nicki Holighaus et al. [7] addresses the problem of phase retrieval when only the magnitude information of a signal is available from a wavelet transform. The authors present a non-iterative approach that uses explicit relationships between the log-magnitudes and the phase gradient of an analytical function referred to as a Cauchy wavelet. The paper reports that when the system is used to approximate phase coefficients, this method is computationally efficient and generalizes well for other mother wavelet functions.

Antiderivative Antialiasing for Stateful Systems [8] by Martin Holters addresses a specific problem in antialiasing, which is that an effective method proposed by Parker et al. [9] using the antiderivative of the integrated amplitude of a time-step in a signal is limited to memoryless systems. The paper suggests modifications to the algorithm, which extend the method to stateful systems. This makes the method particularly useful for modeling some forms of analog circuitry.

Audio Transport: A Generalized Portamento via Optimal Transport by Trevor Henderson and Justin Solomon [10] introduces an approach to creating a portamento effect between any two arbitrary acoustic sources. Portamento is generally an effect which is reserved for instruments that allow constant pitch variation; however the paper solves this problem using a method based on the geometric optimal transport algorithm. The method interpolates partials between two different magnitude spectra using the optimal transport map, and is validated through subjective listening tests on synthesized and natural sounds.

\section{WIMP5}

The Workshop on Intelligent Music Production (WIMP) has been running for five years, and hosts a range of talks on audio signal processing, machine learning, psychoacoustics, and audio engineering. This time it was held in connection with DAFx in the same premises. Two keynotes were included, and six papers were accepted for presentation. In the morning keynote session, Lydia Gregory (FeedForward AI, UK) presented her work on commercializing music technology research. The talk provided insight into the collaborative challenges when integrating creative machine learning systems. In the afternoon session, Vesa Välimäki (Aalto University, Finland) presented his work on audio equalization. The talk provided a summary of equalization methods, and focused on the recent work relating to EQ design using neural networks, which were trained to learn the mapping between target and optimized filter gains at specified center frequencies [11].

In September 2020, the 6th WIMP workshop will be held at the Interdisciplinary Centre for Computer Music Research at the University of Plymouth, UK, and chaired by David Moffat.

\section{Sponsors}

The generous sponsors of DAFx 2019 were NeuralDSP, Soundtoys, Eventide, Sonnox, Arturia, ROLI, FXPansion, iZotope, Antelope Audio, PreSonus, Mathworks, Yamaha, Semantic Audio Labs, UVI, AudioKinetic, and MDPI that furthermore offered publication free of charge for the awarded best papers in Applied Sciences, Special Issue "Digital Audio Effects". We would also like to thank Native Instruments and Ableton for co-funding inclusivity grants.

\section{Conclusions and Prospects}

This meeting report has provided information about the DAFx 2019 conference, which had the same theme as this special issue: digital audio effects. Since this event was a success both scientifically and in terms of number of participants, it is expected that the DAFx conference series will continue annually after this 22nd edition. A particularly positive feature of this conference series is that companies in the audio and music tech area keep sending their representatives to the event. This shows that the interest in this topic is not only academic, but businesses have an urge to learn more about this flourishing research field and to transfer novel ideas from scientific research to their products. 
The next DAFx conference will take place in Vienna, Austria, 8-12 September 2020. As the DAFx conference is a truly international event, but it has been arranged almost every time in Europe, it is the hope of all those involved that volunteers could be found to organize future DAFx conferences in other continents, such as in Asia or in America.

Author Contributions: All authors contributed equally to this publication. All authors have read and agreed to the published version of the manuscript.

Funding: This research received no external funding.

Conflicts of Interest: The authors declare no conflict of interest.

\section{References}

1. Mathew, M.; Grossman, J.; Andreopoulou, A. Women in audio: Contributions and challenges in music technology and production. In Proceedings of the Audio Engineering Society 141th Convention, Los Angeles, CA, USA, 29 September-2 October 2016.

2. Cecchi, S.; Carini, A.; Spors, S. Room response equalization-A review. Appl. Sci. 2017, 8, 1-47. [CrossRef]

3. Werner, K.J. Virtual Analog Modeling of Audio Circuitry Using Wave Digital Filters. Ph.D. Thesis, Stanford University, Stanford, CA, USA, December 2016.

4. Werner, K.J.; Bernardini, A.; Smith, J.O.; Sarti, A. Modeling circuits with arbitrary topologies and active linear multiports using wave digital filters. IEEE Trans. Circ. Syst. I Regul. Pap. 2018, 65, 4233-4246. [CrossRef]

5. Kato, J.; Ogata, M.; Inoue, T.; Goto, M. Songle Sync: A large-scale web-based platform for controlling various devices in synchronization with music. In Proceedings of the ACM Multimedia Conference, Seoul, Korea, 22-26 October 2018; pp. 1697-1705.

6. Kato, J.; Nakano, T.; Goto, M. TextAlive: Integrated design environment for kinetic typography. In Proceedings of the 33rd Annual ACM Conference on Human Factors in Computing Systems, Seoul, Korea, 18-23 April 2015; pp. 3403-3412.

7. Holighaus, N.; Koliander, G.; Abreu, L.D.; Pruša, Z. Non-iterative phaseless reconstruction from wavelet transform magnitude. In Proceedings of the 22nd International Conference on Digital Audio Effects, Birmingham, UK, 2-6 September 2019.

8. Holters, M. Antiderivative antialiasing for stateful systems. In Proceedings of the 22nd International Conference on Digital Audio Effects, Birmingham, UK, 2-6 September 2019.

9. Parker, J.D.; Zavalishin, V.; Le Bivic, E. Reducing the aliasing of nonlinear waveshaping using continuous-time convolution. In Proceedings of the 19th International Conference on Digital Audio Effects (DAFx-16), Brno, Czech Republic, 5-9 September 2016; pp. 137-144.

10. Henderson, T.; Solomon, J. Audio transport: A generalized portamento via optimal transport. In Proceedings of the 22nd International Conference on Digital Audio Effects, Birmingham, UK, 2-6 September 2019.

11. Välimäki, V.; Rämö, J. Neurally controlled graphic equalizer. IEEE/ACM Trans. Audio Speech Lang. Process. (TASLP) 2019, 27, 2140-2149. [CrossRef]

(C) 2019 by the authors. Licensee MDPI, Basel, Switzerland. This article is an open access article distributed under the terms and conditions of the Creative Commons Attribution (CC BY) license (http://creativecommons.org/licenses/by/4.0/). 